\title{
A qualitative study of the experience of returning to family life and the coping styles of patients after total laryngectomy
}

\author{
Hua Yang ${ }^{1 \wedge}$, Fangfang Zeng ${ }^{1}$, Tianying Pang ${ }^{1}$, Hongyu Zhang ${ }^{2}$, Juan Lu ${ }^{1}$ \\ ${ }^{1}$ Department of Otolaryngology-Head and Neck Surgery, Nanfang Hospital, Southern Medical University, Guangzhou, China; ${ }^{2}$ Department of \\ Comprehensive Ward, Zengcheng Branch of Nanfang Hospital, Southern Medical University, Guangzhou, China \\ Contributions: (I) Conception and design: H Yang; (II) Administrative support: J Lu; (III) Provision of study materials or patients: H Yang, J Lu; (IV) \\ Collection and assembly of data: F Zeng, H Zhang; (V) Data analysis and interpretation: H Yang, T Pang; (VI) Manuscript writing: All authors; (VII) \\ Final approval of manuscript: All authors. \\ Correspondence to: Hua Yang. Department of Otolaryngology-Head and Neck Surgery, Nanfang Hospital, Southern Medical University, Guangzhou, \\ China. Email: ebigking@163.com.
}

Background: Total laryngectomy is the major treatment for stage III-IV laryngeal cancer, which prolongs patient's life; however, brings significant changes to physical function, mental health, and social life. As a result, patients must adopt various strategies to cope with these changes and challenges. Few studies have investigated the individual experiences of laryngectomized patients returning to home and society in China. Here we explored the living conditions and inner experiences of laryngectomized patients after discharge from the hospital, and identified the main difficulties they encountered and their coping strategies.

Methods: This study adopted a qualitative descriptive approach with a semi-structured interview. Purposive sampling helped to recruit 19 patients who had undergone total laryngectomy at a tertiary hospital. Patients were interviewed at their follow-up visits 6-12 months after discharge. The interview content was analyzed according to the Creswell steps. Similar descriptions of the patients' experiences were categorized into specific themes.

Results: Three major theme categories were extracted from the real-world experiences of patients following total laryngectomy to return to their former roles in family/social life and work-related activities: (I) impact of surgery on family life, including changes in physical function (e.g., decreased physical activities and functional impairment) and family roles (e.g., becoming a burden to the family and attempts to live as before); (II) impact of surgery on returning to society, including reduced social interaction and difficulty in social reintegration; and (III) the patients' coping styles, including negative coping (e.g., avoidance, compromise, and limited access to social resources) and positive coping (e.g., self-motivation and effective use of social resources).

Conclusions: After total laryngectomy, patients face numerous challenges, and adopt different coping styles to deal with stressful situations. Care and support from family can help them adapt to the change in role and regain confidence in life. Actively seeking social support and using social resources effectively can help patients overcome adverse psychological effects and promote self-identity. Medical staff should provide personalized social support (including information, emotional support, and belonging), and strengthen the post-discharge follow-up and continuous management to promote the functional recovery of patients.

Keywords: Total laryngectomy; return to home; life experience; coping style; qualitative research

Submitted Aug 16, 2021. Accepted for publication Nov 12, 2021.

doi: 10.21037/apm-21-2687

View this article at: https://dx.doi.org/10.21037/apm-21-2687

^ ORCID: 0000-0003-2371-8923. 


\section{Introduction}

Laryngeal cancer is a common malignant tumor of the head and neck, accounting for $1-5 \%$ of systemic malignancies. It ranks third among otolaryngological tumors, after nasopharyngeal cancer and nasal cavity/ paranasal sinus cancers (1). Total laryngectomy is the major treatment modality for stage III-IV laryngeal cancer, and the 5 -year survival rates of patients after surgery are $60-64 \%$ (2). Surgical treatment of laryngeal cancer requires the complete removal of tumor tissues to prolong the lives of patients. However, surgery also destroys the normal anatomy of the larynx, resulting in temporary or permanent physiological and functional changes, including altered airway, loss of voice, difficulty swallowing, taste and smell impairments, and appearance changes due to the tracheostoma, which in turn affect patient mental health and quality of life (3-6). Laryngectomized patients might develop psychological disorders as a negative reaction to the experience of physical, functional, and lifestyle changes, such as anxiety, depression, post-traumatic stress disorder, or alcohol dependence $(4,7)$. Patients need to adopt different strategies to cope with the threat of the disease itself as well as the immediate and long-term impacts of surgical treatment, so as to return to their normal daily life or achieve a new balance between their disease status and real-world life $(6,8)$.

Qualitative research focuses on exploring individuals' perspectives and interpreting the meanings they give to a social or human problem (9). van Sluis et al. focused on the experiences of women following total laryngectomy for at least 1 year to identify their specific rehabilitation needs for psychosocial and practical support to reintegrate into socialand work-related activities (10). The qualitative study conducted by Bickford et al. (11) showed the long-term influence of biopsychosocial, cultural, and linguistic factors on the psychosocial adjustment of laryngectomized patients, as well as their persisting need for psychosocial support. However, little is known about the specific individual problems encountered by this population or their personal coping experiences in their cancer survival journey within the first year after surgery in China, particularly those who could not use tracheoesophageal speech or voice prostheses to communicate.

Therefore, this study aims to qualitatively investigate the living conditions and inner experiences of total laryngectomy patients during the 6-12-month postoperative period, and identify the main difficulties they encountered when returned to their families and social life, and their coping strategies, in an attempt to provide targeted support services in the future. Purposive sampling was used to recruit the participants and a semi-structured interview was selected as the means of data collection in this study. Purposive selection is perceived as an appropriate sampling procedure to help researcher better understand the research question in a particular field (9). The semi-structured interview refers to the verbal questioning of a combination of predetermined questions and follow-up probes, which allows for some flexibility to probe for more information, in order to obtain rich and meaningful data (12).

\section{Methods}

\section{Participants}

This study was conducted in accordance with the Declaration of Helsinki (as revised in 2013) (13) and was approved by the Medical Ethics Committee of Nanfang Hospital of Southern Medical University (No. NFEC2021-272). Patients diagnosed with laryngeal cancer who underwent total laryngectomy in Nanfang Hospital, a tertiary general hospital in Guangzhou, China, from January 2018 to December 2019 were selected as the study population using a purposive sampling method. The inclusion criteria were as follows: (I) newly diagnosed with laryngeal cancer, underwent total laryngectomy, and were discharged with a tracheostomy tube; (II) aged $\geq 18$ years; and (III) signed written consent forms, and could describe their experiences through verbal narration or written text. The exclusion criteria included the following: (I) diagnosed with neurological disorders and/or cognitive impairment or other conditions that could affect their participation; and (II) unable to speak, read, and/or write. The final sample size was determined by data saturation; that is, when no new themes emerged from the data analysis. Finally, 19 patients were interviewed and their demographic data are summarized in Table 1.

\section{Data collection}

A qualitative descriptive approach was adopted in current study. Based on the inclusion criteria, researchers contacted patients who had undergone total laryngectomy at our center from January to October 2018 by mail. From November 2018 to December 2019, patients were invited to participate in the study while they were still in the 
Table 1 Demographic data of the interviewees $(n=19)$

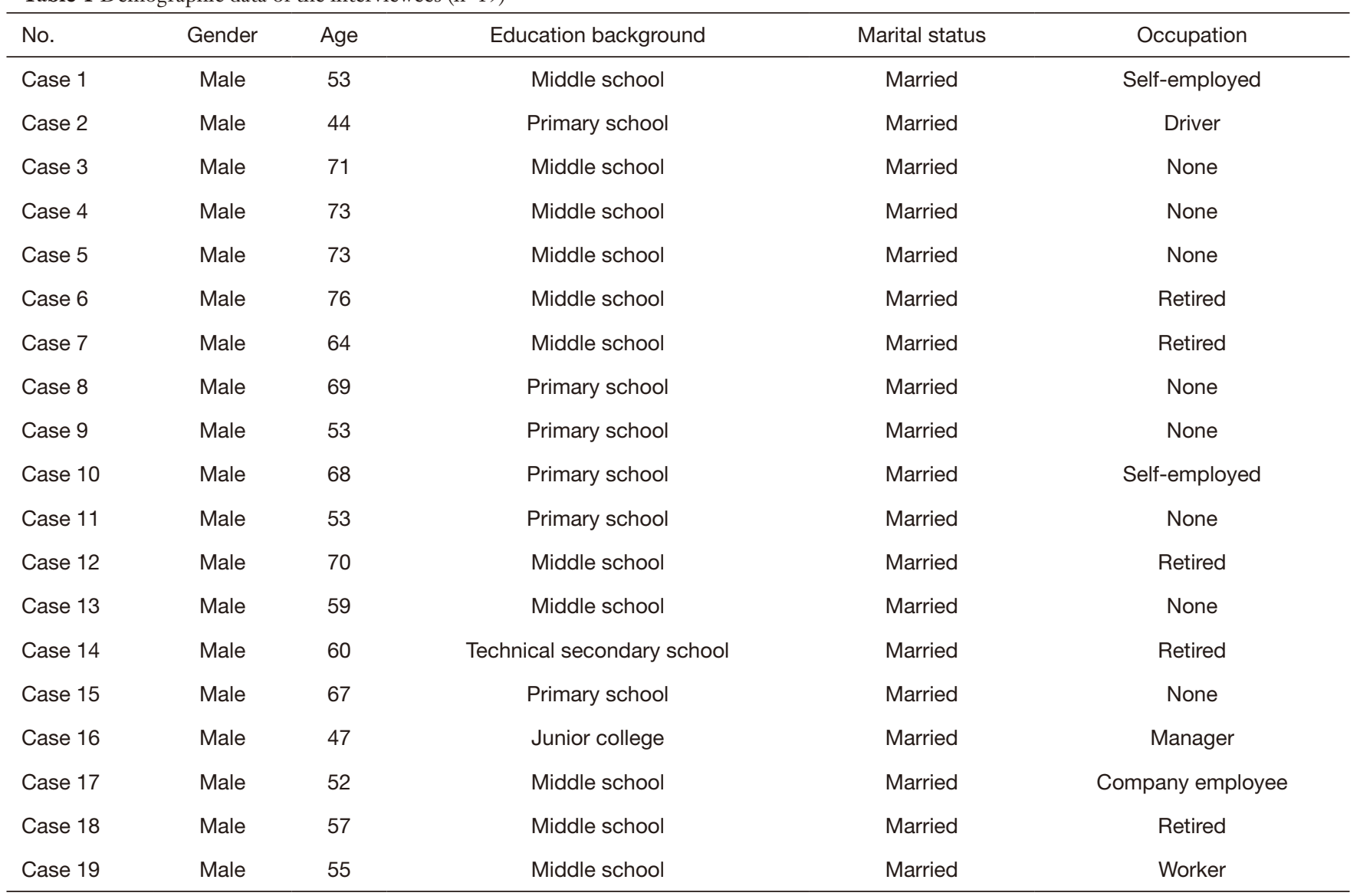

hospital, supplying them with information about the study. Patients who agreed to participate were interviewed during their outpatient visits (6-12 months after discharge) in a quiet and comfortable consultation room, avoiding outside distractions. The aim of the study, the content of the interview, the confidentiality of personal data, the rights of patients, and possible risks were again explained prior to the interview. The patients were informed that they could request to stop the interview at any time. The interviewees voluntarily signed an informed consent form.

A clinical nurse specialist conducted a semi-structured interview with the patient. A topic guide was used to frame the interview based on previous studies and the specialist knowledge of the research team, beginning with an openended question: "Can you tell me what your life has been like since discharge?" The interview included the following questions: "How did the surgery affect your life after going home?", "What were the greatest difficulties you encountered?", "How did you resolve it?", "What are your thoughts about your physical recovery?", "Have your roles in your family changed?", "How has your social life changed?", "Have you received help from social groups or individuals after discharge from the hospital?", "How did you assess and use these social supports?", "Did your health care provider give you enough guidance about your life after discharge?", "What kind of help would you like?" Follow-up questions for each topic were added and adapted based on the patients' responses, allowing respondents to elaborate as much as possible on their experiences. For post-total laryngectomy patients who could communicate via tracheoesophageal speech or voice prostheses, the entire interview process was recorded with a digital voice recorder; meanwhile, for patients who could not speak, the questions were answered by narrative writing. Some patients were accompanied by a family member who was living with them. An experienced research assistant attended the interview and took field notes, including the interviewee's emotions, feelings, and body language, as well as some relevant information provided by the family members, which had been confirmed by the interviewees on the spot. 


\section{Data analysis}

Qualitative data collected on the digital recorders and written notes during the interview were identified and transcribed verbatim by two researchers who organized the collected information and returned it to the interviewees via WeChat, in order to verify the precision of the transcription and clarify the meaning. The collected data were analyzed according to the steps proposed by Creswell et al. (9). First, the researchers read all transcribed text and field notes to obtain an overall view of the research questions. The researcher then extracted important statements (i.e., units of meaning) from each interview and progressively coded the data, which involves aligning data, creating segments, creating categories, and labeling categories. NVivo 11 Plus was used to code, store, and retrieve the data. During the coding process, these statements were grouped together and themes were developed around similar statements.

\section{Results}

In this study, 19 patients were interviewed and the interview time ranged from 30 to 60 minutes, with an average of 40 minutes. The physical and psychological experiences of each post-total laryngectomy patient after returning to home and society differed, and the following three theme categories were summarized.

\section{Impact of surgery on patients' return to family life}

\section{Changes in physical function}

Total laryngectomy in patients with laryngeal cancer affected basic physiological functions, including eating and swallowing. Although most patients could already eat through the mouth when discharged from the hospital, the food intake gradually changed from a full fluid diet to a semi-fluid diet and then soft food, which, when combined with surgery trauma, could easily cause a physical decline in patients. Case 6: "I do not want to exercise. I live in a four-story bouse. Now I live on the $1^{\text {st }}$ floor and I feel out of breath when I walk upstairs. I have to stop when I walk. And shopping is not as long as before." Case 9: "Since I had surgery, my shoulders burt when I turn around at night. My sleep quality is very poor; sometimes I cannot sleep all night. My body feels very strained without doing anything, and my physical fitness has decreased a lot."

Surgery also led to impairment of smell and taste, as well as difficulty in movement of the limbs at the surgical site. Case 13: "Recently, my right shoulder hurts and it is diffcult to lift my right hand, so I flip my arms to move my shoulders when I walk, and the pain will be slightly alleviated. My doctor told me that I had my right cervical lymph nodes removed, but did not perform shoulder exercises after surgery, so it had an impact." Case 19: "My physical strength is worse than before surgery. I cannot lift heavy items, I cannot smell, and my taste is worse than before. Wounds on my right arm, shoulder, and neck still cause numbness."

\section{Change in family roles}

After the patient became ill, the original family lifestyle was disrupted, and the family needed to devote more energy to the patient's care. The patients were also unable to continue working or taking care of their families due to the disease. They could not assume their original family and social roles, and some still required the care of family members. Patients felt that they had become a burden to their families. Case 11: "In the past, I was the main breadwinner of my family. I used to be a bricklayer. However, I cannot work anymore after surgery, and the family income depends mainly on the children." Case 5: "My health is not as good as before after surgery, and now I live with my daughter. The tracheostomy tube must be disinfected daily. My daughter helps me in doing so. I do not know bow to do it."

In contrast, some patients adapted to the change of role immediately after returning home and tried to resume to their former life status. Case 12: "I received surgery about a year ago. Today, I usually walk, exercise, play mahjong, help the family with grocery shopping, cooking, and fishing. Life has not changed much from before." Case 19: "Now I help take care of the children at home and complete the scheduled pick-ups and drop-offs. I usually go grocery shopping, cook, and watch TV." Case 18: "After being discharged from the hospital, my life has basically returned to what it was before. I change the pads and wash the tracheostomy tube myself, and I can take care of myself completely. I applied for retirement before surgery. I have been a believer in Buddhism for 5 years. I usually listen to and chant sutras at home and live a peaceful life, taking things as they come."

\section{Impact of surgery on patients' return to social life}

\section{Reduced social interaction}

Most of the interviewees reported that they were reluctant to communicate with others or go out because they could not express themselves clearly after total laryngectomy. Case 13: "The problem is that I cannot talk on the phone and communication with friends is not very convenient (especially 
since I cannot have phone conversations). When friends come to visit, I sometimes open my mouth to talk but cannot speak clearly. My wife helps me to 'interpret' because she understands my 'language' better. If I cannot speak clearly, I use my cell phone to communicate by WeChat. Handwriting can be clearly expressed, but the communication is still less than before." Case 8: "Before surgery, I liked to make friends and chat with my neighbors. After the surgery, I cannot speak. When I see my friends, I nod or use other gestures. I cannot make new friends anymore."

\section{Social reintegration}

Some interviewees also stated that they actively reintegrated into their original social circles after discharge from hospital. Case 14: "I usually love to fish and chat with friends. My colleagues and classmates often come to visit, and everyone cares about me." Case 16: "I have many old friends. After the surgery, my colleagues and friends understand my condition and are concerned about me, and they do not look at me differently." Case 18: "I usually communicate with my friends who also believe in Buddhism through WeChat to share ideas. Sometimes I meet with them to worship in a nearby temple. My parishioners are caring, understanding, and inclusive, and do not discriminate against me because I am sick."

\section{Coping styles}

After total laryngectomy, patients encounter the threat of the disease itself (e.g., the fear of disease recurrence) as well as the distress caused by their altered appearance, swallowing difficulties, and communication problems postoperatively. Coping strategies vary among patients after they return home and may change during adaptation.

\section{Negative coping \\ Avoidance and compromise}

During the initial period of returning to their families and communities, most of the patients showed social withdrawal, low self-esteem, and impatience. They avoided or reduced communication and exchanges with others. Case 1: "The impact of the surgery was huge when I got home. I did not dare go out on the street. I had low self-esteem. I could not talk. I had very little communication with my friends. I tried to avoid it, as others would look at me with different eyes." Case 7: "I am still troubled by the change in my appearance. I feel embarrassed when I eat in front of other people, but it is okay with my family. Some people worry if the disease is contagious, so they will avoid me." Case 19: "I have some difficulty in eating, talking to people, and socializing with friends, and I feel a little embarrassed in public places."

Due to communication difficulties, patients could not accurately express their needs, and often dealt with the changes negatively, such as impatience, irritability or compromise. Case 8: "Not being able to speak is the biggest challenge, and the first few months after discharge were a living hell for me. I would get impatient and lose my temper when I was uncomfortable, and it took 2 or 3 months to ease up." Case 6: "I used to be a teacher, but now I communicate with my family through writing, which is not as convenient as before. Sometimes it is difficult to make they understand what I want, so I give up and do not mention it."

\section{Limited access to social resources}

Due to insufficient awareness of the disease, patients attempted to continuously obtain more information related to treatment and rehabilitation after returning home, usually through consulting medical personnel, communicating with other patients, and using the Internet. However, verbal communication difficulties restricted patients with a low educational level in accessing and utilizing informational resources. Case 2: "I had surgery to save my life and I did not worry too much about speaking at that time, and I did not bother to learn about it when I was discharged from the hospital. When I got home, I wanted to learn new ways of pronunciation, but I did not know where I could learn." Case 4: "I want to speak a lot and I am ready to buy an electronic larynx, but there is no information about it. I do not know where to buy it and I also do not know how to receive training." Case 11: "There is a similar group of post-laryngectomy patients in my hometown, but it is far away, and I did not participate in their activities or contact them. My daughter joined a WeChat group sponsored by your department and would consult the group about pronunciation training and follow-up visits. When she saw other patients mentioning uncomfortable symptoms, she would ask me if I had similar problems and learn how to deal with them." Case 5: "I live in Chongqing. A few days ago, I wanted to take a followup examination in a nearby hospital, and the doctor told me that I should be examined at the hospital where I had the surgery. In my bometown, there is no laryngectomized patient group that can belp."

\section{Active coping \\ Self-motivation}

Total laryngectomy means a lifetime loss of normal articulatory function. Some patients adapted to physical and functional changes and promoted self-identity through selfmotivation and persistent exercise. Case 16: "At first, I was a little worried about the way I look now, but my wife bought 
me a black crushed silk scarf, which keeps out the dust from the stoma and also looks fashionable. I would wrap it around my neck when I went out, which makes me quite good-looking." "I wake up at 7 a.m., exercise for 1 hour in the morning, and take a walk for another bour in the afternoon. So my life bas become very regular, and I'm in good spirits during the day." Case 1: "When I was discharged from the hospital, I could not pronounce words, so I was very pessimistic, and sometimes I really wanted to give up. Although I was equipped with an electronic larynx and my communication with family members was okay, I was embarrassed by my special voice when talking in public and the special eyes of other people. One day I went fishing and a frog kept croaking next to me. An idea came to me, 'Even frogs can talk, why can't I?' In the coming days, I practiced repeatedly and finally learned to pronounce with my esophagus. Now I want to start a New Voice club to carry out charity activities to belp more people without larynx and teach them the esophageal speech faceto-face." Case 2: "I was and am the breadwinner of my family. I became a driver after my physical condition improved. Since I needed to talk to customers, I tried to practice pronunciation and slowly I could express myself more clearly, but only face-to-face."

\section{Effective use of social resources}

As shown in the interviews, some patients actively sought other social support and made full use of the department's communication platform, aiming to overcome adversity and support a positive emotional experience. Case 12: "I was quite scared and depressed after the surgery. The medical staff invited patients who had undergone the same surgery to visit me, during which they spoke to me with an electronic larynx and told me to relax and take it easy. One of the patients even gave me his electronic larynx to try out and told me how to find the focal point. Under his guidance, I learned to use the electronic larynx to make sounds and felt much better. Six months after I was discharged, my daughter bought me an electronic larynx, and now I am quite familiar with it." "Now I am a member of The New Voice Club of Hong Kong. They send me materials regularly, which are quite helpful." Case 18: "The first time I knew that I could still speak after this surgery was when I attended the charity event 'Regaining New Voice' beld by your hospital, and from then on, I was determined to learn to speak without the electronic larynx. After about 2 months of practice, I could already communicate with people in esophageal speech."

\section{Discussion}

Coping refers to an individual's conscious, purposeful, and flexible regulatory behavior in response to changes in the real environment (14). Despite successfully removing the lesion, total laryngectomy also destroys some of the patients' innate basic physiological functions, causing a huge impact on their mental status, emotions, lifestyle, family relationships, and social functions. Patients and their family members must adopt various coping strategies to facilitate full recovery and improve their quality of life. Previous studies have shown that different patients adopt different coping styles, which directly or indirectly affect the prognosis and outcome (15). Adopting active or proactive coping strategies (e.g., positive cognitive behaviors and actively seeking and using social support) can help laryngectomized patients address stress properly and maintain a healthy psychological state. Conversely, negative and passive coping strategies (e.g., avoidance or distancing) are often associated with negative psychology, including anxiety and depression, and poorer prognosis and quality of life (15-17).

\section{Communication impairment and appearance change are the main dilemmas faced by patients with total laryngectomy}

Our respondents reported that they were mostly concerned about the changes in somatic sensations after surgery at the early stage of going home. They often felt weak and easily fatigued within 1-2 months after discharge, and would suffer from changes in physiological functions (e.g., swallowing, smell, and taste), as well as neck and shoulder pain. As their physical condition gradually improves and their basic survival needs are met, patients' willingness to return to society and resume daily social activities increases. Respondents reported that communication impairment and appearance changes were the main challenges they needed to face during the 6-12-month postoperative period. Therefore, patients may have interpersonal difficulties and impaired social functioning, exhibiting social withdrawal, isolation, and even depression $(7,11)$.

Anterior cervical tracheostoma makes the appearance of patients different to the healthy population, and patients become more sensitive to outsiders' perceptions. Öztürk et al. reported that total laryngectomy patients were disturbed by the tracheostoma, unpleasant odor, and coarse breath sounds, which affected their social activities (5). The formation of permanent tracheostoma creates a sense of stigma. Patients feel that they may be labeled, discriminated against, stereotyped, and not understood or accepted, and therefore fear that the stoma will be discovered. As a result, they will minimize outdoor activities and engage in self- 
isolation (18). In our current study, some interviewees expressed that they felt that "people would especially look at them" and avoided interacting with others for fear of being discriminated against. Total laryngectomy deprives patients of normal articulatory functions, and patients often cannot express their personal feelings and needs in a timely and accurate manner through non-verbal communication, including gestures and words. Some interviewees showed impatience, irritability, and anxiety due to poor communication with their families upon returning home, and adopted negative coping strategies, such as avoidance and compromise.

\section{Family support plays a key role in belping patients cope with role change}

All our respondents agreed that the understanding, support, and encouragement from their families, relatives, and friends motivated them to accept the loss of physical function and re-seek pleasure or meaning in life. After being discharged from the hospital, where the medical staff provided direct and professional care, the patients returned to their families. The physical and physiological functional changes caused by the surgery shifted the focus of their lives and temporarily prevented them from assuming their earlier family roles. With the need for tracheostoma care, patients became more dependent on their families in terms of daily living and finances.

Some patients were less able to adapt to their new roles and felt less secure after returning home; they were prone to anxiety and even depression. Keszte et al. found that approximately $22 \%$ of patients developed anxiety, depression, post-traumatic stress disorder, and other psychiatric problems 1 year after total laryngectomy (4). Family support at this stage plays a crucial role and affects the survival of cancer patients. In a survey of cancer survivors, Osazuwa-Peters et al. found that the suicide rate among survivors of head and neck malignancies was approximately twice as high as other cancer survivors. Although improvements in treatment have led to increased survival rates among cancer patients, the risk of suicide remained a concern for survivors of head and neck malignancies (19). Zhou et al. showed that improving family adaptability, cohesion, and support could help eliminate suicidal ideation in cancer patients (20). Good family support was also beneficial in reducing the incidence of mental disorders, including anxiety and depression, in cancer patients $(21,22)$. Three of our respondents reported that they had suffered enormous hardships and even considered giving up on life due to the significant difference in their roles before and after the disease. When asked how they coped with these thoughts, they replied that their families' understanding, tolerance, and support helped them through the most difficult period, regain confidence in life, and slowly adapt to their home life after surgery.

Patients discharged from the hospital often require long-term and continuous care and support from their caregivers, increasing the burden of the family. Improving patients' self-care skills helps to boost their self-confidence and self-esteem. Twelve of our interviewees were fully able to complete self-care at the time of the interview, while the remaining seven patients were still being cared for in whole or in part by their family members. Medical staff should encourage patients to actively participate in self-care during the postoperative recovery period. For example, staff should instruct patients to wipe any sputum independently in front of the mirror after coughing, and to remove and disinfect the tracheostomy tube, so that they realize that they can still take care of themselves after surgery and do not need to rely excessively on their family members. After returning home, patients may help with certain household chores, such as grocery shopping, cooking, and pick-ups and dropoffs of kids. As a result, they will feel that their families still need them, which helps instill a higher sense of existence, reduce negative emotions such as "being a burden to the family," enhance their self-identity, and encourage them to reintegrate into their families with a more positive attitude.

\section{Early social support helps patients cope positively with the transition}

Social support refers to moral or material support and encourages individuals to accept support from various sources, including society and family, during stressful situations (23). As an external resource, social support plays an important role in helping cancer patients cope with challenges and adapt to different anti-cancer treatment and recovery. The social support system of patients usually includes family, friends, medical staff, social workers, and social groups $(6,24)$. An earlier study revealed that insufficient social resources available and accessible to total laryngectomy patients, and these patients were further limited in accessing and using new support resources due to verbal communication difficulties (25).

As shown in our current study, social support for total laryngectomy patients after returning to their families was 
provided by medical staff and other patients with similar experiences. Patients usually obtain more information about treatment and rehabilitation by consulting medical personnel, communicating with other patients, and using the Internet. There was insufficient identification and use of other types of social support. Patients with total laryngectomy lacked health awareness and knowledge and had limited access to relevant information. Some patients hesitated to seek other types of social support. Most of our interviewees lived in towns or communities that lacked organizations for laryngectomized patients, and local grassroots health care providers also lacked knowledge of these patients, making it difficult to supply the information or home services that the patients needed.

Our interviews also revealed that earlier supportive interventions (i.e., during hospitalization) focusing on the difficulties after returning home could help patients cope with changes in physical function after surgery, adapt to family life more quickly, and reintegrate into society with a more positive attitude. Tailored health education by nurses before discharge can improve patients' participation in self-care after discharge (26). For example, nurses can instruct patients and their family members on the methods and precautions for home airway care by sending a video to caregivers. In addition, a WeChat group for total laryngectomy patients may be established to learn and help deal with problems encountered at home, creating a sense of belonging and reducing anxiety caused by the insufficient routine follow-up care offered by healthcare professionals. Peer support can also improve patient prognoses (27). Asking patients with the same surgical experiences to present themselves in the preoperative or early postoperative period allows patients to see themselves in the future and increase their confidence in overcoming the disease. Some of our interviewees reported that they participated in the hospital's public welfare activities for laryngectomized patients during hospitalization. The sharing of peers greatly encouraged them to actively cooperate with treatment. They took the initiative to participate in rehabilitation training (including esophageal speech and use of the electronic larynx) upon completion of treatment, and learned effective verbal communication methods more quickly.

\section{Implications and recommendations for clinical practice}

The current study interviewed total laryngectomy patients during the 6-12-month post-operative period to focus on their initial transition from hospital to home. The descriptive method helped to reflect the actual "voice" of each laryngectomized patient, which contributed to better understanding the ways that this population reacted and responded to the challenges they confronted. This study highlighted the important issues and needs from the patient's perspective via exploring their living conditions, inner experiences, and coping mechanisms after returning home.

Medical staff typically tends to pay attention to the psychological status of cancer patients during hospitalization, but often ignores emotional support in the continuing care. This study exposed the possible mental and emotional problems of total laryngectomy patients after discharge. The medical staff should analyze the possible causes and provide corresponding psychological nursing measures to patients during pre-discharge education and follow-up, in order to help patients maintain a good psychological condition and promote treatment of the disease and rehabilitation.

After returning to home and society, laryngectomized patients require professional guidance on tracheostoma care, esophageal speech training, and swallowing function training, etc. To improve their quality of life, providing professional, comprehensive and timely continuing nursing service for this population is essential. The development of the "Internet + nursing service" model (28), which extends nursing services from the hospital to the community/ home, is conducive to meeting this need. Furthermore, an integrated hospital-community-family network management service platform (29) can promptly resolve the physical and mental problems of patients, improve family coping ability, and promote the recovery of patients.

Peer support has been shown to be of great importance in the rehabilitation of laryngectomized patients. Besides holding peer education and public welfare activities for laryngectomy patients regularly, medical staff may use modern communication and teaching methods, such as micro-lecture videos and online patient education, to help patients understand and learn relevant skills and enhance their confidence in coping with postoperative problems.

\section{Conclusions}

In summary, total laryngectomy patients exhibit different coping mechanisms upon returning to home and society as a result of their physical and functional changes, including changes in appearance, loss of voice, and altered taste and 
smell. Care and support from family and friends can help patients adapt to role changes and regain confidence in life as soon as possible. Actively seeking social support and effectively using social resources can assist patients in overcoming adverse psychological effects and promote self-identity. As the main supporters of patients, medical personnel should provide tailored social support, including information, emotional support, and belonging. Continuity of care may be offered by promoting the "Internet + nursing service" model and building network management platforms like "Hospital-Community-Family Trinity". By regularly conducting public welfare activities for laryngectomized patients, medical personnel can strengthen mutual support among patients to promote functional rehabilitation and improve their quality of life. As a result, patients can return to society and face their futures positively.

Our current study had some limitations that should be noted. Firstly, all of the subjects were males, and there were insufficient real-life experiences of women who had total laryngectomy living at home. Secondly, most of the interviewees were unable to express themselves verbally, and thus, data were collected mainly in writing. Due to the varying levels of literacy, some patients may not have been able to express their true feelings because they could not fully write, and the richness of the data could be somewhat affected. Thirdly, family members accompanied some patients during the interviews, which could have affected the patients' full expression and information biases.

\section{Acknowledgments}

Funding: This study was financially supported by the Nursing Research Fund of Southern Medical University (Y2018006). The funding source was not involved in the design, intervention, data collection and analysis, and publication of this study.

\section{Footnote}

Data Sharing Statement: Available at https://dx.doi. org/10.21037/apm-21-2687

Conflicts of Interest: All authors have completed the ICMJE uniform disclosure form (available at https://dx.doi. org/10.21037/apm-21-2687). All authors report that this study was financially supported by Nursing Research Fund of Southern Medical University (Y2018006). The authors have no other conflicts of interest to declare.
Ethical Statement: The authors are accountable for all aspects of the work in ensuring that questions related to the accuracy or integrity of any part of the work are appropriately investigated and resolved. This study was conducted in accordance with the Declaration of Helsinki (as revised in 2013). The study was approved by the Medical Ethics Committee of Nanfang Hospital of Southern Medical University (No. NFEC-2021-272). The interviewees voluntarily signed an informed consent form.

Open Access Statement: This is an Open Access article distributed in accordance with the Creative Commons Attribution-NonCommercial-NoDerivs 4.0 International License (CC BY-NC-ND 4.0), which permits the noncommercial replication and distribution of the article with the strict proviso that no changes or edits are made and the original work is properly cited (including links to both the formal publication through the relevant DOI and the license). See: https://creativecommons.org/licenses/by-nc-nd/4.0/.

\section{References}

1. Chen $\mathrm{W}$, Zheng R, Zhang S, et al. Cancer incidence and mortality in China, 2013. Cancer Lett 2017;401:63-71.

2. Shen LF, Zhou SH, Yu Q. Relationships between expression of glucose transporter protein-1 and hypoxia inducible factor-1 $1 \alpha$, prognosis and 18F-FDG uptake in laryngeal and hypopharyngeal carcinomas. Transl Cancer Res 2020;9:2824-37.

3. Guibert M, Lepage B, Woisard V, et al. Quality of life in patients treated for advanced hypopharyngeal or laryngeal cancer. Eur Ann Otorhinolaryngol Head Neck Dis 2011;128:218-23.

4. Keszte J, Danker H, Dietz A, et al. Mental disorders and psychosocial support during the first year after total laryngectomy: a prospective cohort study. Clin Otolaryngol 2013;38:494-501.

5. Öztürk A, Mollaoğlu M. Determination of problems in patients with post-laryngectomy. Scand J Psychol 2013;54:107-11.

6. Dooks $\mathrm{P}, \mathrm{McQuestion} \mathrm{M}$, Goldstein D, et al. Experiences of patients with laryngectomies as they reintegrate into their community. Support Care Cancer 2012;20:489-98.

7. Bussian C, Wollbrück D, Danker H, et al. Mental health after laryngectomy and partial laryngectomy: a comparative study. Eur Arch Otorhinolaryngol 2010;267:261-6.

8. Chen PY, Chang HC. The coping process of patients with cancer. Eur J Oncol Nurs 2012;16:10-6. 
9. Creswell JW. Research Design. Thousand Oaks: SAGE Publications, 2013.

10. van Sluis KE, Kornman AF, van der Molen L, et al. Women's perspective on life after total laryngectomy: a qualitative study. Int J Lang Commun Disord 2020;55:188-99.

11. Bickford J, Coveney J, Baker J, et al. Living with the altered self: a qualitative study of life after total laryngectomy. Int J Speech Lang Pathol 2013;15:324-33.

12. Kader P. Nursing Research Principle, Process and Issue (Second edition). Palgrave Macmillan, 2006.

13. World Medical Association. World Medical Association Declaration of Helsinki: ethical principles for medical research involving human subjects. JAMA 2013;310:2191-4.

14. Joffe PE, Bast BA. Coping and defense in relation to accommodation among a sample of blind men. J Nerv Ment Dis 1978;166:537-52.

15. Eadie TL, Bowker BC. Coping and quality of life after total laryngectomy. Otolaryngol Head Neck Surg 2012;146:959-65.

16. Aarstad AK, Lode K, Larsen JP, et al. Choice of psychological coping in laryngectomized, head and neck squamous cell carcinoma patients versus multiple sclerosis patients. Eur Arch Otorhinolaryngol 2011;268:907-15.

17. Horney DJ, Smith HE, McGurk M, et al. Associations between quality of life, coping styles, optimism, and anxiety and depression in pretreatment patients with head and neck cancer. Head Neck 2011;33:65-71.

18. Danielsen AK, Soerensen EE, Burcharth K, et al. Impact of a temporary stoma on patients' everyday lives: feelings of uncertainty while waiting for closure of the stoma. J Clin Nurs 2013;22:1343-52.

19. Osazuwa-Peters N, Simpson MC, Zhao L, et al. Suicide risk among cancer survivors: Head and neck versus other cancers. Cancer 2018;124:4072-9.

Cite this article as: Yang H, Zeng F, Pang T, Zhang H, Lu J. A qualitative study of the experience of returning to family life and the coping styles of patients after total laryngectomy. Ann Palliat Med 2021;10(11):11482-11491. doi: 10.21037/apm-21-2687
20. Zhou Y, Hu D, Zhang K, et al. The mechanism of family adaptability and cohesion in suicidal ideation among Chinese cancer patients. J Psychosoc Oncol 2020;38:612-26.

21. Lekka D, Pachi A, Tselebis A, et al. Pain and Anxiety versus Sense of Family Support in Lung Cancer Patients. Pain Res Treat 2014;2014:312941.

22. Su JA, Yeh DC, Chang CC, et al. Depression and family support in breast cancer patients. Neuropsychiatr Dis Treat 2017;13:2389-96.

23. Pedersen AF, Olesen F, Hansen RP, et al. Social support, gender and patient delay. Br J Cancer 2011;104:1249-55.

24. Semple CJ, McCance T. Experience of parents with head and neck cancer who are caring for young children. J Adv Nurs 2010;66:1280-90.

25. Taylor A, Wells M, Hubbard G, et al. From an illusion of certainty into a reality of uncertainty: A longitudinal qualitative study of how people affected by laryngeal cancer use information over time. Eur J Oncol Nurs 2016;23:15-23.

26. Dobbins M, Gunson J, Bale S, et al. Improving patient care and quality of life after laryngectomy/glossectomy. $\mathrm{Br}$ J Nurs 2005;14:634-40.

27. Brodar KE, Carlisle V, Tang PY, et al. Identification and Characterization of Peer Support for Cancer Prevention and Care: A Practice Review. J Cancer Educ 2020:1-10.

28. Huang R, Xu M, Li X, et al. Internet-Based Sharing Nurse Program and Nurses' Perceptions in China: CrossSectional Survey. J Med Internet Res 2020;22:e16644.

29. Fanghua G, Sanhui T. Internet Intervention System for Elderly Hypertensive Patients Based on Hospital Community Family Edge Network and Personal Medical Resources Optimization. J Med Syst 2020;44:95.

(English Language Editors: J. Chapnick and A. Kassem) 